\title{
Severe Hypoglycemia in Hospitalized Children with Diabetic Ketoacidosis in Brazzaville
}

\author{
Aymar Pierre Gildas Oko*, Judicael Kambourou, Engoba Moyen, Laetitia Lombet, \\ Neli Yvette Ngakengni, Steve Vassili Missambou Mandilou, Aurore Mbika Cardorelle, \\ Georges Marius Moyen
}

Faculty of Health Sciences, Marien Ngouabi University of Brazzaville, Brazzaville, Congo

Email: *aymaroko@yahoo.fr

How to cite this paper: Oko, A.P.G., Kambourou, J., Moyen, E., Lombet, L., Ngakengni, N.Y., Mandilou, S.V.M., Cardorelle, A.M. and Moyen, G.M. (2020) Severe Hypoglycemia in Hospitalized Children with Diabetic Ketoacidosis in Brazzaville. Open Journal of Pediatrics, 10, 236-245.

https://doi.org/10.4236/ojped.2020.101024

Received: December 18, 2019

Accepted: March 24, 2020

Published: March 27, 2020

Copyright $\odot 2020$ by author(s) and Scientific Research Publishing Inc. This work is licensed under the Creative Commons Attribution International License (CC BY 4.0).

http://creativecommons.org/licenses/by/4.0/

(c) (i) Open Access

\begin{abstract}
Background: Severe hypoglycemia is a lifethreatening iatrogenic complication in the management of diabetic ketoacidosis, however, few studies have described how often this complication occurs. Objective: To identify the risk factors for severe hypoglycemia in children treated for diabetic ketoacidosis and to describe the sociodemographic, clinical characteristics and outcome of these children. Methods: From January 2013 to December 2017, 69 children admitted to pediatric intensive care unit with a diagnosis of diabetic ketoacidosis were enrolled in a retrospective and prospective study. Data of children with and without severe hypoglycemia were compared. Data were analyzed using Epi Info 7.1.5.0. Results: Out of 69 children enrolled, 10 (14.5\%) had severe hypoglycemia (girls: 60\%, median age 144 months (interquartile range, 108 - 168 months)). Episodes of severe hypoglycemia occurred during the first 48 hours of hospitalization in $8(80 \%)$ children and during the late night shift in $6(60 \%)$ children. Causes of severe hypoglycemia were: excessive insulin dose $(60 \%)$, inappropriate infusion solution $(70 \%)$, failure to properly monitor blood glucose levels (40\%). Severe hypoglycemia risk factors were: age $<5$ years $(p=0.00004$; OR $=42.0$ [95\% CI: $6.3-279])$ and severe malnutrition $(p=0.002$; OR $=10.9$ [95\% CI: $2.4-48.6])$. Of 8 (11.6\%) children died, 4 had severe hypoglycemia ( $p=0.01$; OR $=8.8$ [95\% CI, $1.7-44.8]$ ). Conclusion: The importance and severity of severe hypoglycemia require preventive measures to control risk factors.
\end{abstract}

\section{Keywords}

Severe Hypoglycemia, Diabetic Ketoacidosis, Risk Factors, Brazzaville

\section{Introduction}

Diabetic ketoacidosis is a common and severe medical complication seen in di- 
abetic children. Its frequency varies from $15 \%$ to $70 \%$ depending on regions and countries and in The Republic of Congo, it is $30 \%$ to $79 \%$ [1] [2] [3]. Despite steady improvements in patients care, diabetic ketoacidosis remains a major cause of morbidity and mortality in diabetic children, especially in Africa [3] [4].

Severe hypoglycemia is often cited as one of diabetic ketoacidosis's iatrogenic complications that may contribute to the critical morbidity and mortality cited above [5] [6]. However, not so many researches have been devoted to this condition and, the few data available are related to adults patients [7] [8]. Severe hypoglycemia in diabetic children is usually the result of excessive insulin supply on one hand and, insufficient carbohydrates intake and/or inadequate counter-regulatory hormone response on the other hand. It has been mainly studied in ambulatory settings [9] [10]. It is worth noting that, improved insulin quality, the insulin therapy and state of the art blood glucose monitoring systems have all contributed to a general decreased of its incidence worldwide [9]. These same measures coupled with a timely glucose intake have shown the same effect on the incidence of hypoglycemia during diabetic ketoacidosis [5] [11]. However, some authors still report cases of hypoglycemia in children while in care for diabetic ketoacidosis, and sometimes in significant numbers [12] [13] [14]. Unfortunately, these studies do not provide enough information regarding severe hypoglycemia; the fundamental reason is not well understood: One wonders if the scarcity is lack of cases, under recording or rather a matter simple trivialization? In Brazzaville (Republic of Congo), a previous study of children treated for diabetic ketoacidosis reported the frequency of severe hypoglycemia at $27.2 \%$ [3]. Understanding factors associated with such a high frequency should help implement measures and strategies that are geared toward improving the care of Congolese children with diabetic ketoacidosis and consequently, reducing the frequency of this condition.

The main purpose of this study was to identify the risk factors associated with hypoglycemia in children treated for diabetic ketoacidosis and to determine the sociodemographic, clinical, characteristics and outcome of these children.

\section{Methods}

\subsection{Setting, Design and Population of Study}

From January 2013 to December 2017, we conducted a retrospective and prospective analytical study in the Pediatric Intensive Care Unit (PICU) of University Teaching Hospital (UTH) of Brazzaville.

Children hospitalized for diabetic ketoacidosis were systematically included.

Children with diabetic ketoacidosis who died upon admission and those whose records did not provide sufficient information were excluded from the study.

\subsection{Study Procedures}

Upon admission in the PICU, children received medical care follow the recom- 
mended protocols of the International Society for Pediatric and Adolescent Diabetes (ISPAD) [15] [16].

These protocols involve, a continuous intravenous insulin therapy at $0.05-0.1$ $\mathrm{UI} / \mathrm{kg} / \mathrm{h}$, following at least 1 to 2 hours of fluid replacement therapy, an adjusted insulin dosage correlating to patients' blood glucose levels, a glucose supply when blood glucose levels are between $14-17 \mathrm{mmol} / \mathrm{L}(2.5-3.0 \mathrm{~g} / \mathrm{L})$ or when glucose level rapidly falls ( $>5 \mathrm{mmol} / \mathrm{L} / \mathrm{h}$ ), blood glucose readings on admission and every hour during the period of intravenous insulin therapy. Blood glucose monitoring was performed every 2 - 4 hours during subcutaneous insulin therapy.

Blood glucose measurements were performed using an Accuchek ${ }^{\otimes}$ brand of capillary blood glucose monitors. A laboratory measurement of plasma glucose was performed to confirm hypoglycemia cases diagnosed with the glucometer. Patients' families were the responsible for providing the accessories such as test strips for blood glucose readings as well as the necessary medications.

For each child, data related to: sex, age, socio-economic status, personal and family history of diabetes, nutritional status, level of consciousness assessed using the Glasgow coma scale [17], blood glucose readings on admission and during hospitalization, insulin dosage administered on admission and during hospitalization, type intra venous (IV) fluids used, the outcome (discharge, death), were all collected using a standardized pre-established survey form by way of interviewing the parents/legal guardians and the child him/herself when possible and from medical records.

\subsection{Operational Definitions}

Diabetic Ketoacidosis was diagnosed using standard definitions [16]. Hypoglycemia [18]:

$\checkmark$ Clinical hypoglycemia alert: was defined as a blood glucose value $\leq 3.9$ $\mathrm{mmol} / \mathrm{L}(0.7 \mathrm{~g} / \mathrm{L})$.

$\checkmark$ Clinically critical or serious hypoglycemia: blood glucose value $<3 \mathrm{mmol} / \mathrm{L}$ $(0.54 \mathrm{~g} / \mathrm{L})$.

$\checkmark$ Severe hypoglycemia: hypoglycaemic event leading to neurocognitive impairments, loss of consciousness or seizure unrelated to another cause. And in the case of neurocognitive disorders related to another cause, blood glucose value $<2.75 \mathrm{mmol} / \mathrm{L}(0.5 \mathrm{~g} / \mathrm{L})$ was used to define severe hypoglycemia.

\subsection{Statistical Analysis}

Data were collected, processed and analyzed using the software Epi Info 7.1.5.0. The quantitative variables were expressed as mean \pm standard deviation or median and interquartile range (IQR). The qualitative variables were expressed as percentages. The number of each variable was also specified. To identify the risk factors for severe hypoglycemia, we performed a univariate analysis, since the small sample of children with severe hypoglycemia did not allow for a multivariate analysis. The comparison of percentages was performed using the inde- 
pendence chi square test or the Fischer test (when at least one of the theoretical numbers was less than 5) and the odds ratio. The comparison of means was carried out using the Analysis of Variance (ANOVA) or with the Mann Whitney-Wilcoxon test. The significance level was set at $5 \%$ and the confidence interval at $95 \%$.

\subsection{Ethical Considerations}

For each child an informed consent was obtained from parents or legal guardians. The study was conducted in compliance with the Helsinki Declaration and approved by the National Committee of Ethics in Health Sciences.

\section{Results}

\subsection{Characteristics of the Study Population}

During the study period, a total of 8860 children were hospitalized, and admitted to the PICU. Among them 74 were hospitalized for ketoacidosis, 5 were excluded because they did not fulfil the required criteria ( 2 died upon arrival and 3 had incomplete files), therefore, 69 were selected for our study. There were 46 girls (66.7\%) and 23 boys (33.3\%), the median age of the study population was 144 months (IQR, 108 - 168 months). Mean blood glucose on admission was $26.4 \mathrm{mmol} / \mathrm{L} \pm 5.2$ (extreme, 14.9 and 35.8 ).

Twenty-six (37.7\%) children had hypoglycemia, among them 17 (24.6\%) had clinical hypoglycemia alert, 13 (18.6\%) clinically important hypoglycemia and 10 (14.5\%) severe hypoglycemia; some children had different types of hypoglycemia.

\subsection{Severe Hypoglycemia}

Among the 10 children with severe hypoglycemia, $70 \%$ were female. The female to male ratio was 1.1, children aged under 5 years represented $60 \%$ of cases; Sociodemographic, clinical and paraclinical characteristics of children hospitalized for diabetic ketoacidosis according to the presence or absence of severe hypoglycemia are shown in Table 1.

The mean blood glucose level during the episodes of severe hypoglycemia was $2.1 \mathrm{mmol} / \mathrm{L} \pm 0.44$ (extremes: 1.1 and 2.5).

Eight children (80\%) presented episodes of severe hypoglycemia during the first 48 hours of hospitalization and while on insulin infusion.

Episodes of severe hypoglycemia occurred in 6 children (60\%) during the late night shift (between 12:00 AM and 4:00 AM), in 2 at the end of the day shift (between 6:00 PM - 7 PM) and 2 in the morning shift (between 7:00 AM and 11:00 AM).

Neurocognitive signs manifested during episodes of severe hypoglycemia were uniquely related consciousness status: Glasgow score at 12 and $13(n=7)$, Glasgow score at $10(\mathrm{n}=2)$ and Glasgow score at $6(\mathrm{n}=1)$.

The identified causes that contributed to the severe hypoglycemia were: Non adjusted infusion solution or insulin posology to blood glucose respectively 7 
Table 1. Sociodemographic, clinical and paraclinical characteristics of children hospitalized for diabetic ketoacidosis according to the presence or absence of severe hypoglycemia.

\begin{tabular}{|c|c|c|c|c|}
\hline Characteristics & $\begin{array}{c}\text { Severe } \\
\text { hypoglycemia }\end{array}$ & & $O R[95 \% C I]$ & $P$-value \\
\hline & No & Yes & & \\
\hline & $\mathrm{n}=59$ & $\mathrm{n}=10$ & & \\
\hline Median age (months) [IQR] & $156[138-186]$ & $36[12-134]$ & & $0.0006^{*}$ \\
\hline \multicolumn{5}{|l|}{ Gender } \\
\hline \multirow{2}{*}{$\begin{array}{l}\text { Female } \\
\text { Male }\end{array}$} & $39(66.1)$ & $7(70.0)$ & \multirow[b]{2}{*}{$0.8[0.19-3.5]$} & \multirow[b]{2}{*}{0.8} \\
\hline & $20(33.9)$ & $3(30.0)$ & & \\
\hline \multicolumn{5}{|l|}{ SES } \\
\hline High & $5(8.5)$ & $0(0.0)$ & \multirow{3}{*}{$0.2[0.01-1.4]$} & \multirow{3}{*}{0.06} \\
\hline Middle & $22(37.3)$ & $1(10.0)$ & & \\
\hline Low & $32(54.2)$ & $9(90.0)$ & & \\
\hline \multicolumn{5}{|l|}{ Personal history of diabetes } \\
\hline No & $35(59.3)$ & $9(90.0)$ & \multirow{2}{*}{$0.1[0.01-1.36]$} & \multirow{2}{*}{0.06} \\
\hline Yes & $24(40.7)$ & $1(10.0)$ & & \\
\hline \multicolumn{5}{|l|}{ Severe malnutrition } \\
\hline No & $51(86.4)$ & $4(40.0)$ & \multirow{2}{*}{$9.5[2.2-41.5]$} & \multirow{2}{*}{$0.003^{*}$} \\
\hline Yes & $8(13.6)$ & $6(60.0)$ & & \\
\hline \multicolumn{5}{|l|}{ GCS $<11$} \\
\hline No & $41(69.5)$ & $7(70.0)$ & \multirow{2}{*}{$0.9[0.2-4.2]$} & \multirow{2}{*}{0.9} \\
\hline Yes & $18(30.5)$ & $3(30.0)$ & & \\
\hline $\begin{array}{l}\text { Mean blood glucose on } \\
\text { admission } \pm \text { SD }\end{array}$ & $28.6 \pm 7.1$ & $31.3 \pm 7.1$ & & 0.4 \\
\hline
\end{tabular}

$O R$ : odds ratio; $C I$ : confidence interval; $I Q R$ : intervalle interquartile; SES: socioeconomic status; GCS: Glasgow coma scale; SD: standard deviation; ${ }^{*}$ : difference statistically significant.

(70\%) and $6(60 \%)$ children, last but not least, the failure to properly monitor blood glucose levels in 4 children (40\%).

\subsection{Severe Hypoglycemia Risk Factors (Table 2)}

Severe hypoglycemia risk factors were: age $<5$ years $(p=0.00004$; OR $=42.0$ [IC: 6.3 - 279]) and severe malnutrition ( $p=0.002$; OR $=10.9$ [IC: $2.4-48.6])$.

\subsection{Outcome}

The median duration of hospital stay was 7 days [IQR, 4.5 - 10.5] in children who did not have severe hypoglycemia and 6 days [IQR, 6 - 12] in those who presented the condition $(p=0.9)$. Of 69 children, $61(88.4 \%)$ survived and 8 (11.6\%) died. Four of the 8 deceased children had severe hypoglycemia ( $p=0.01$; $\mathrm{OR}=8.8$ [IC, $1.7-44.8]$ ).

The causes of death were: shock $(n=4)$, cerebral edema $(n=2)$, severe hypoglycemia $(\mathrm{n}=1)$ and severe hypokalemia $(\mathrm{n}=1)$.

\section{Discussion}

This study, a first of its kind in Congo, allowed us to determine the incidence of severe hypoglycemia in children treated for ketoacidosis and to identify the 
Table 2. Risk factors of severe hypoglycemia.

\begin{tabular}{|c|c|c|c|c|}
\hline \multirow[t]{2}{*}{ Characteristics } & \multicolumn{2}{|c|}{ Severe hypoglycemia } & \multirow[t]{2}{*}{$O R[95 \% C I]$} & \multirow[t]{2}{*}{$P$-value } \\
\hline & $\begin{array}{c}\text { No } \\
n=59\end{array}$ & $\begin{array}{c}\text { Yes } \\
n=10\end{array}$ & & \\
\hline \multicolumn{5}{|l|}{ Age $<$ ans } \\
\hline Yes & 2 & 6 & \multirow{2}{*}{42.0 [IC: 6.3 - 279] } & \multirow{2}{*}{$0.00004^{*}$} \\
\hline No & 56 & 4 & & \\
\hline \multicolumn{5}{|l|}{ Severe malnutrition } \\
\hline No & $51(86.4)$ & $4(40.0)$ & \multirow{2}{*}{$9.5[2.2-41.5]$} & \multirow{2}{*}{$0.003^{*}$} \\
\hline Yes & $8(13.6)$ & $6(60.0)$ & & \\
\hline \multicolumn{5}{|l|}{ GCS $<11$} \\
\hline No & $41(69.5)$ & $7(70.0)$ & \multirow{2}{*}{$0.9[0.2-4.2]$} & \multirow{2}{*}{0.9} \\
\hline Yes & $18(30.5)$ & $3(30.0)$ & & \\
\hline
\end{tabular}

associated risk factors. The incidence (14.5\%) observed in this work is lower than that (27.2\%) reported in a previous study [3], nevertheless, it is still too high for such a serious and easily avoidable complication. The decrease in the incidence recorded in this work can be attributed to an improved care and management of these children. The worldwide incidence of severe hypoglycemia in children treated for ketoacidosis is poorly known because the condition has not been widely studied. The few studies that reported cases of hypoglycemia in children treated for ketoacidosis used different definitions and often did not specify the proportion of severe hypoglycemia [12] [13] [14]. It is therefore difficult to compare our results with those reported by these authors. However, some of the reported data in these studies may be informative; in India, Jayashree and al., using a definition of hypoglycemia (BG $<0.5 \mathrm{~g} / \mathrm{L})$ similar to our second definition of severe hypoglycemia, but without including the neurocognitive impairment status did observed hypoglycemia in $15 \%$ of children treated for diabetic ketoacidosis [19]. In contrast, Peeters et al. in England, observed only $3.2 \%$ of cases of hypoglycemia (blood glucose $<3 \mathrm{mmol} / \mathrm{L}$ ) [20]. The incidence of clinically important hypoglycemia (glycemia $<3.0 \mathrm{mmol} / \mathrm{L}$ or $0.54 \mathrm{~g} / \mathrm{L}$ [18]) appears to be more frequent in developing countries than in developed; the same may be true for severe hypoglycemia. Peeters et al. explain their observed low incidence of hypoglycemia by the use of a lower standard dose of insulin $(0.03-0.05 \mathrm{IU} / \mathrm{kg} / \mathrm{h})$ versus $(0.05-0.1 \mathrm{IU} / \mathrm{kg} / \mathrm{h})$ the one usually recommended by most experts [5] [6], and the use of solute containing glucose from the beginning of insulin therapy. Several studies have shown that the use of insulin at doses $<0.05 \mathrm{IU} / \mathrm{Kg} / \mathrm{h}$ could prevent the sudden fall in blood glucose and consequently prevent the occurrence of hypoglycemia unlike the usage of doses $\geq 0.05$ $\mathrm{IU} / \mathrm{Kg} / \mathrm{h} \mathrm{[21]} \mathrm{[22].} \mathrm{Although} \mathrm{it} \mathrm{is} \mathrm{recognized} \mathrm{that} \mathrm{continuous} \mathrm{intravenous} \mathrm{insulin}$ therapy at a dosage of $0.5-1 \mathrm{IU} / \mathrm{Kg} / \mathrm{h}$ has contributed to the reduction in the incidence of hypoglycemia during treatment of diabetic ketoacidosis [5] [11], it appears that the use of lower insulin doses $(0.03-0.05 \mathrm{IU} / \mathrm{Kg} / \mathrm{h})$ could further reduce it. In the present study, the use of insulin doses $0.05-0.1 \mathrm{IU} / \mathrm{kg} / \mathrm{h}$ in all cases, might have contributed to the high incidence of severe hypoglycemia. 
However, the true identified causes of severe hypoglycemia were: failure to adjust carbohydrate intake and insulin dosage in accordance to blood glucose levels and the failure to consistently monitor blood glucose levels. The difficulties for some poor parents to ensure a regular supply of drugs and blood glucose for monitoring test strips explain the weaknesses in care and management observed. This situation is a reality in many countries where health care costs are patients' responsibilities and universal health care coverage is nonexistent. However, the inability of health care staff to ensure optimal supervision of all patients during the night shift can also be considered as another reason; indeed, severe hypoglycemia episodes occurred mainly during the second part of the night shift, a period during which fatigue is more pronounced and the medical team is reduced in numbers, thus with a higher workload. The other possible explanation for the severe hypoglycemia episodes during the second part of night is the decrease response of counter regulatory system to hypoglycemia during sleep, a phenomenon responsible for nocturnal hypoglycemia in diabetic subjects. The prevalence of nocturnal hypoglycemia can be as high as $25 \%$ in diabetic children [23].

In our study, severe hypoglycemia episodes occurred in $80 \%$ of cases during the first 48 hours of hospitalization. For Jayashree and al. all these episodes happened before 36 hours [19]. In both studies, this time period corresponds to the period of intravenous insulin therapy, a period with high risk of hypoglycemia due to relatively high daily insulin doses administered. The multiple blood glucose measurements done during this period may also explain why more severe hypoglycemia episodes were discovered.

The risk factors of severe hypoglycemia in this study were age $<5$ years and severe malnutrition. In their study, Jayashree and al. had found a predominance of hypoglycemia (blood glucose $<0.5 \mathrm{~g} / \mathrm{L}$ ) in severely malnourished children, and Moulik in all malnourished children [19] [24]. These children are initially more exposed to hypoglycemia due to a lower carbohydrate reserve [25]. It would be wise in those cases to start insulin therapy with lower insulin doses $(0.03-0.05 \mathrm{IU} / \mathrm{Kg} / \mathrm{h})$ and to implement a more rigorous blood glucose monitoring scheme for an early detection and correction of a risky situation. Continuous glucose monitoring systems would be of great help in this regard [26].

Lethality in this study was greater in the group of children with severe hypoglycemia. Hypoglycemia's responsibility in the death of diabetic children has been reported by several authors [27]. Severe hypoglycemia would lead to death through fatal cardiac rhythm disorders, multiple visceral organs failures and primary brain death [28]. Severe Hypoglycemia would also play a role in the dead in bed syndrome; a sudden deaths of young healthy patients with type 1 diabetes that happens at night without signs of agitation nor loss of urine and without sweating [29]. In this study, only one death was directly related to severe hypoglycemia, the other death in children with severe hypoglycemia happened during out the diagnostic period, since these children because of their fragility (young age, severe malnutrition) presented other serious complications such as septic shock, cerebral edema, hypokalemia that lead to fatal outcome. 
Limitations of study: this study had some limitations. The first was the fact of not being able to perform a multivariate analysis of the risk factors due the small size/sample of the children population with severe hypoglycemia, hence, limiting the power of comparative tests used. Nevertheless, the study made it possible for the first time in Congo and perhaps in Africa, to specify the incidence of this complication and to identify the risk factors. The second limitation was the retrospective collection of some of the data used, what may raise the issue of the reliability of these data.

\section{Conclusion}

This study showed that severe hypoglycemia was a common complication in children treated for diabetic ketoacidosis in Brazzaville, and exposed them to an increased mortality. The main circumstances that contribute to its occurrence can be avoided with a meticulous respect of the guidelines and a better organization of care. The severely malnourished children and those under 5 years who are the most at risk will need a more rigorous supervision.

\section{Conflicts of Interest}

The authors declare no conflicts of interest regarding the publication of this paper.

\section{References}

[1] Usher-Smith, J.A., Thompson, M., Ercole, A. and Walter, F.M. (2012) Variation between Countries in the Frequency of Diabetic Ketoacidosis at First Presentation of Type 1 Diabetes in Children: A Systematic Review. Diabetologia, 55, 2878-2894. https://doi.org/10.1007/s00125-012-2690-2

[2] Dabelea, D., Rewers, A., Stafford, J.M., et al. (2014) Trends in the Prevalence of Ketoacidosis at Diabetes Diagnosis: The SEARCH for Diabetes in Youth Study. Pediatrics, 133, 938-945. https://doi.org/10.1542/peds.2013-2795

[3] Oko, A.P.G., Ali, F.K.Z., Missambou Mandilou, S.V., et al. (2018) Diabetic Ketoacidosis in Children: Epidemiological and Prognostic Aspects. The Pan African Medical Journal, 31, 167. https://doi.org/10.11604/pamj.2018.31.167.14415

[4] Peer, N., Kengne, A.P., Motala, A.Y. and Mbanya, J.C. (2014) Diabetes in the African Region: An Update. Diabetes Research and Clinical Practice, 103, 197-205. https://doi.org/10.1016/j.diabres.2013.11.006

[5] Dunger, D.B., Sperling, M.A., Acerini, C.L., et al. (2004) ESPE/LWPES Consensus Statement on Diabetic Ketoacidosis in Children and Adolescents. Archives of Disease in Childhood, 89, 188-194. https://doi.org/10.1136/adc.2003.044875

[6] Wolfsdorf, J.I., Glaser, N., Agus, M., et al. (2018) ISPAD Clinical Practice Consensus Guidelines 2018: Diabetic Ketoacidosis and the Hyperglycemic Hyperosmolar State. Pediatric Diabetes, 19, 155-177. https://doi.org/10.1111/pedi.12701

[7] Fischer, K.F., Lees, J.A. and Newman, J.H. (1986) Hypoglycemia in Hospitalized Patients, Causes and Outcomes. The New England Journal of Medicine, 315, 1245-1250. https://doi.org/10.1056/NEJM198611133152002

[8] Malone, M.L., Klos, S.E. and Gennis, V.M. (1992) Frequent Hypoglycemic Episodes 
in the Treatment of Patients with Diabetic Ketoacidosis. Archives of Internal Medicine, 152, 2472-2477. https://doi.org/10.1001/archinte.1992.00400240090015

[9] Bulsara, M.K., Holman, C.D., Davis, E.A. and Jones, T.W. (2004) The Impact of a Decade of Changing Treatment on Rates of Severe Hypoglycemia in a Population-Based Cohort of Children with Type 1 Diabetes. Diabetes Care, 27, 2293-2298. https://doi.org/10.2337/diacare.27.10.2293

[10] Birkebaek, N.H., Drivvoll, A.K., Aakeson, K., et al. (2017) Incidence of Severe Hypoglycemia in Children with Type 1 Diabetes in the Nordic Countries in the Period 2008-2012: Association with Hemoglobin A1c and Treatment Modality. BMJ Open Diabetes Research \& Care, 5, e000377. https://doi.org/10.1136/bmjdrc-2016-000377

[11] Burghen, G.A., Etteldorf, J.N., Fisher, J.N. and Kitabchi, A.Q. (1980) Comparison of High-Dose and Low-Dose Insulin by Continuous Intravenous Infusion in the Treatment of Diabetic Ketoacidosis in Children. Diabetes Care, 3, 15-20. https://doi.org/10.2337/diacare.3.1.15

[12] Lopes, C.L., Pinheiro, P.P., Barberena, L.S. and Eckert, G.U. (2017) Diabetic Ketoacidosis in a Pediatric Intensive Care Unit. Jornal de Pediatria, 93, 179-184. https://doi.org/10.1016/j.jped.2016.05.008

[13] Kuppermann, N., Ghetti, S., Schunk, J.E., et al. (2018) Clinical Trial of Fluid Infusion Rates for Pediatric Diabetic Ketoacidosis. The New England Journal of Medicine, 378, 2275-2287. https://doi.org/10.1056/NEJMoa1716816

[14] Onyiriuka, A. and Ifebi, E. (2013) Ketoacidosis at Diagnosis of Type 1 Diabetes in Children and Adolescents: Frequency and Clinical Characteristics. Journal of Diabetes and Metabolic Disorders, 12, 47. https://doi.org/10.1186/2251-6581-12-47

[15] Wolfsdorf, J., Craig, M.E., Daneman, D., Dunger, D., Edge, J., Lee, W., Rosenbloom, A., Sperling, M. and Hanas, R. (2009) Diabetic Ketoacidosis in Children and Adolescents with Diabetes. Pediatric Diabetes, 10, 118-133. https://doi.org/10.1111/j.1399-5448.2009.00569.x

[16] Wolfsdorf, J.I., Allgrove, J., Craig, M.E., Edge, J., Glaser, N., Jain, V., Lee, W.W.R., Mungai, L.N.W., Rosenbloom, A.L., Sperling, M.A. and Hanas, R. (2014) A Consensus Statement from the International Society for Pediatric and Adolescent Diabetes: Diabetic Ketoacidosis and Hyperglycemic Hyperosmolar State. Pediatric Diabetes, 15, 154-179. https://doi.org/10.1111/pedi.12165

[17] Jennett, B. and Teasdale, G. (1977) Aspects of Coma after Severe Head Injury. The Lancet, 1, 878-881. https://doi.org/10.1016/S0140-6736(77)91201-6

[18] Abraham, M.B., Jones, T.W., Naranjo, D., et al. (2018) ISPAD Clinical Practice Consensus Guidelines 2018: Assessment and Management of Hypoglycemia in Children and Adolescents with Diabetes. Pediatric Diabetes, 19, 178-192. https://doi.org/10.1111/pedi.12698

[19] Jayashree, M. and Singhi, S. (2004) Diabetic Ketoacidosis: Predictors of Outcome in a Pediatric Intensive Care Unit of a Developing Country. Pediatric Critical Care Medicine, 5, 427-433. https://doi.org/10.1097/01.PCC.0000137987.74235.5E

[20] Peeters, E., van Ijperen, W.J., Robertson, L., Royle, P. and van IJperen, W.J.Sr. (2015) Analysis of the Safety and Efficacy of Diabetic Ketoacidosis Management in a Community General Hospital, 2001-2010: A Descriptive Study. Scottish Medical Journal, 60, 121-125. https://doi.org/10.1177/0036933015592291

[21] Bradley, P. and Tobias, J.D. (2007) Serum Glucose Changes during Insulin Therapy in Pediatric Patients with Diabetic Ketoacidosis. American Journal of Therapeutics, 14, 265-268. https://doi.org/10.1097/01.mjt.0000209687.52571.65 
[22] Nallasamy, K., Jayashree, M., Singhi, S. and Bansal, A. (2014) Low-Dose vs Standard-Dose Insulin in Pediatric Diabetic Ketoacidosis: A Randomized Clinical Trial. JAMA Pediatrics, 168, 999-1005. https://doi.org/10.1001/jamapediatrics.2014.1211

[23] Wilson, D.M., Calhoun, P.M., Maahs, D.M., et al. (2015) Factors Associated with Nocturnal Hypoglycemia in At-Risk Adolescents and Young Adults with Type 1 Diabetes. Diabetes Technology \& Therapeutics, 17, 385-391.

https://doi.org/10.1089/dia.2014.0342

[24] Moulik, N.R., Jayashree, M., Singhi, S., et al. (2012) Nutritional Status and Complications in Children with Diabetic Ketoacidosis. Pediatric Critical Care Medicine, 13, e227-e333. https://doi.org/10.1097/PCC.0b013e31823c9a11

[25] Bandsma, R.H., et al. (2010) Mechanisms behind Decreased Endogenous Glucose Production in Malnourished Children. Pediatric Research, 68, 423-428.

https://doi.org/10.1203/PDR.0b013e3181f2b959

[26] Battelino, T., Phillip, M., Bratina, N., Nimri, R., Oskarsson, P. and Bolinder, J. (2011) Effect of Continuous Glucose Monitoring on Hypoglycemia in Type 1 Diabetes. Diabetes Care, 34, 795-800. https://doi.org/10.2337/dc10-1989

[27] Cryer, P.E.H.S. (2012) Severe Hypoglycaemic Predicts Mortality in Diabetes. Diabetes Care, 35, 1814-1816. https://doi.org/10.2337/dc12-0749

[28] Novodvorsky, P., Bernjak, A., Chow, E., et al. (2017) Diurnal Differences in Risk of Cardiac Arrhythmias during Spontaneous Hypoglycemia in Young People with Type 1 Diabetes. Diabetes Care, 40, 655-662. https://doi.org/10.2337/dc16-2177

[29] Tu, E., Twigg, S.M. and Semsarian, C. (2010) Sudden Death in Type 1 Diabetes: The Mystery of the "Dead in Bed" Syndrome. International Journal of Cardiology, 138, 91-93. https://doi.org/10.1016/j.ijcard.2008.06.021 